\title{
The Tycho-2 Catalogue: a reference for astrometry and photometry. Combining the most modern and the oldest astrometric data
}

\author{
C. Turon
}

\begin{abstract}
GEPI-UMR CNRS 8111, Observatoire de Paris, Section de Meudon, 92195 Meudon Cedex, France
e-mail: catherine.turon@obspm.fr
\end{abstract}

The main Hipparcos mission, the first astrometric satellite ever launched, was included in the ESA Science Programme in March 1980. It was aiming at the fundamental task of measuring the angular positions and displacements of about 100000 pre-selected stars to obtain a large set of distances and proper motions that are more accurate and homogenous than ever before and that drastically improve our knowledge of the structure, kinematics, and dynamics of the local part of our Galaxy and of the various types of stars present there (see for example Turon \& Arenou 2008). The resulting catalog was published in 1997 (Perryman et al. 1997), and it indeed opened the way to a wide variety of astrophysical applications (Perryman 2009).

In the project as approved by ESA in 1980, the function of the star mapper was to provide data for determining the realtime satellite attitude, as required to reach the expected accuracy on the astrometric parameters of the 118000 program stars. In March 1981, the ideas of introducing two-color channels in the star mapper and using the continuous flow of star mapper data in survey mode for astrometric and two-color photometric measurements of all stars down to about 10-11 mag was proposed by Høg (Høg et al. 1982). This enhancement was formally approved by ESA a few months later, and the Tycho data analysis consortium (TDAC) was set up in 1982 under the responsibility of Høg. Analysis of this data stream led to the Tycho Catalogue (Høg et al. 1997), a purely observational catalog. Later, the same data stream was reduced again, benefiting of more powerful computers and of the completed Hipparcos Catalogue. Moreover, a major improvement was made by combining the Tycho positions with older astrometric catalogs leading to a strong increase in the accuracy of the proper motions. These accurate proper motions combined with the homogeneity of its photometric observations led to the use of this Tycho-2 Catalogue (Høg et al. 2000) for a wide variety of applications, which would not have been possible with the original catalog.

The Tycho Catalogue, later called "Tycho-1", was compiled from the photon counts recorded by the Hipparcos star mappers. It includes astrometric and two-color photometric parameters for slightly more than one million stars brighter than $V \sim 11.5$ and is $99.9 \%$ complete up to $\sim 10.0$ (Høg et al. 1997). While providing lower accuracies than Hipparcos in the astrometric parameters and only for brighter stars, it has the main advantage of being a survey, using an input catalog only in the first stages of the data analysis, and of providing two-color photometry for all targets.

The Tycho-2 Catalogue was constructed after the mission by combining a new analysis of Tycho data and previous astrometric catalogs. Even though it is based on exactly the same observations as for the original catalog, it is much improved: a new, more powerful, reduction technique was used to analyze the photon counts, resulting in a much larger catalog (2.5 million stars) with deeper completeness $(99.9 \%$ to $\sim 11.0)$ and improved accuracy. Indeed, it also benefited from the final Hipparcos Catalogue and from an improved satellite attitude and instrument calibration. Moreover, the proper motions included in the Tycho-2 Catalogue were no longer computed only from the star mapper data but by combining the Tycho- 2 positions with older, and even much older, positions from 143 transit circle and photographic catalogs. It is very interesting to note that the first international and coordinated effort toward measuring stellar positions, the Astrographic Catalogue (mean epoch 1905), was used here in conjunction with the most modern data provided by the first astrometric satellite.

The resulting accuracy of proper motions, in addition to the very homogeneous two-color photometry, makes Tycho-2 a much used astrometric and photometric reference catalog.

The citations of the Høg et al. paper describing the Tycho2 Catalogue are very numerous (total citations: 587 at the end of March 2009, among which 537 refereed papers), and the history of citations, as given in the ADS database, is quite interesting because it shows a nearly constant rate of citation over the last six years at a level of about 60 citations per year. As a comparison, the citations of the Hipparcos Catalogue are about five times more numerous and show a nearly constant rate of citation over the past eleven years. The other interesting indicator of how the catalog has been used are the statistics of monthly requests addressed to the Tycho-2 Catalogue on all VizieR mirrors (Ochsenbein et al. 2000), from 2006, as shown in Fig. 1. It is impressive to see that the number of interrogations is increasing, with a maximum of 600000 and an average of 230000 per month. These numbers can be compared to similar numbers for the main Hipparcos Catalogue (Perryman et al. 1997): $\max 940000$, average 280000 , and for the UCAC2 Catalogue (Zacharias et al. 2004), which is the astrometric reference catalog for stars down to magnitude 16: $\max 220000$, 


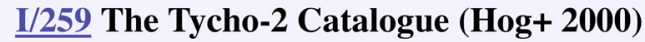

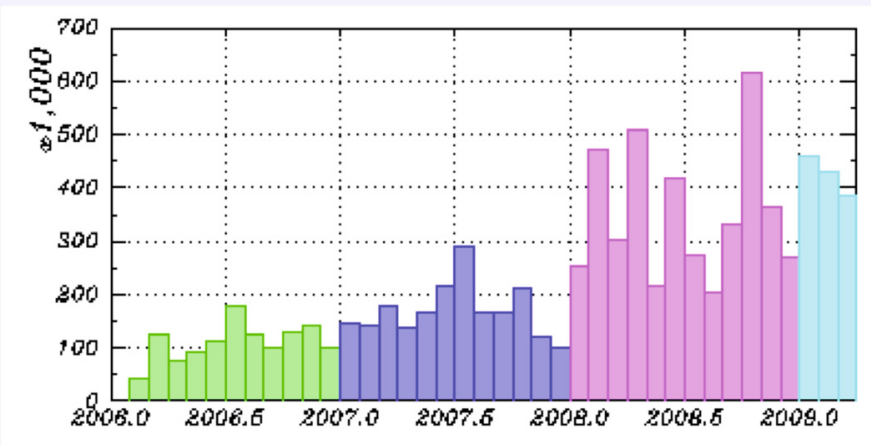

Fig. 1. Monthly requests addressed to Tycho-2 Catalogue on all VizieR mirrors, from 2006.

average 63000 . Another noticeable effect is that the interrogation statistics of these three catalogs exhibit very similar behavior with a parallel and strong increase from 2008. This may be related to the introduction of a description of VizieR in Wikipedia at the end of October 2007, widening the public using VizieR facilities. Of course, this does not take into account the uses of the Hipparcos and Tycho-2 Catalogues directly from the dedicated ESA web page, where multi-parameter search tools are available online.

The uses of the Tycho-2 Catalogue are extremely varied: a small proportion of purely astrometric or catalog compilation applications and a wide variety of astrophysical applications, using the proper motions and the photometry equally, very often in conjunction with the trigonometric parallaxes from the Hipparcos Catalogue or from the new reduction of the raw Hipparcos data performed by van Leeuwen \& Fantino (2005): kinematics of clusters, associations, moving groups, binary and individual stars; detection of binary stars by the comparison of the Tycho-2 and Hipparcos proper motions (the Tycho-2 proper motions being derived from observations covering a much longer time span than the Hipparcos ones); estimation of the spectral type from the Tycho two colors; cluster/association membership; characterization of stars hosting planets; identification of sources detected in X- UV- or IR-rays; variability surveys, etc.

The performance of a space survey for astrometry and photometry is underlined by the history and use of the Tycho- 2 Catalogue. Had it not been part of the Hipparcos project, such a project would very probably never have been accepted in any space science program. Nevertheless, the all-sky accessibility, along with a very stable environment, provide the conditions for much more accurate and homogeneous data than from the ground and has led to an impressive variety of astronomical and astrophysical applications.

\section{Remarks by E. Høg}

It is immensely rewarding that the Tycho-2 Catalogue has been such a success. On behalf of all participants in Tycho, I acknowledge the support we have received from ESA, the Hipparcos Science Team, and all national funding authorities.
It seems appropriate to outline the history of the photoelectric techniques used for the observations. The basic observation technique of Hipparcos was the counting of photoelectrons released from photocathodes by the light of stars crossing slit systems in the focal plane of Hipparcos. In fact, only one star could be measured at a time by each of the one-channel detectors: an image-dissector tube for the main field and two photo-multiplier tubes serving the star mapper channels in the colors $B$ and $V$. The development of photoelectric astrometry began with experiments by Bengt Strömgren in 1925 (see Hфg 2009), using analogue amplification of the photo current. The photon counting was introduced by Hфg (1960). This technique was outdated in the 1990s by CCDs, which could integrate on many stars simultaneously and had much higher quantum efficiency than photocathodes.

The idea of using the star mapper signal to detect other than the 60000 reference stars occurred to me in 1978, but I did not find time to work out the performance. When I did so in March 1981 and realized the enormous potential it was almost too late. Enhancements to an approved mission are usually out of the question for reasons of cost and complexity. But I immediately wrote three reports to the Hipparcos Science Team where the idea was enthusiastically supported. The technical impact of the proposal was to extend the length of the star mapper slits, to include channels for two colors, $B$ and $V$, instead of only one, and to transmit all photon counts to the ground.

The Tycho experiment as initially described (Hog et al. 1982) was expected to measure 400000 stars with accuracies at $B=$ 10 mag of 0.03 arcsec for the positions and 0.03 mag for the $B$ and $V$ magnitudes. Proper motions would be obtained by means of the Astrographic Catalogue with an accuracy of 0.003 arcsec/year. A combined treatment of all available field crossings of each star from a large input catalog would give better accuracy and additionally 800000 detected stars, but this was impossible with the available computing power.

The expectations of 1982 were surpassed in the final Tycho2 Catalogue since 2.5 million stars were obtained and the median accuracies at $B=10$ were about 0.02 arcsec and 0.03 mag, and 0.0015 arcsec/year for the proper motions at that magnitude. There were five reasons for a better performance for faint stars than predicted in 1982: twice as long star mapper slits, higher quantum efficiency, lower sky background, a better method for estimating the background, and the combined treatment of all transits.

\section{References}

Høg, E. 1960, Astron. Abh. der Hamb. Sternw., V, 263

Høg, E. 2009, Development of photoelectric astrometry including the Hipparcos mission, ed. J. Andersen, J. Bland-Hawthorn, \& B. Nordström, Proc. of IAU Symp. 254, and at http://www . astro.ku.dk/ erik/History.pdf Høg, E., Jaschek, C., \& Lindegren, L. 1982, ESA SP-177, 21

Høg, E., Bässgen, G., Bastian, U., et al. 1997, A\&A, 323, L57

Høg, E., Fabricius, C., Makarov, V. V., et al. 2000, A\&A, 355, L27

Ochsenbein, F., Bauer, P., \& Marcout, J. 2000, A\&AS, 143, 221

Perryman, M. A. C. 2009, Astronomical Applications of Astrometry (Cambridge: Cambridge University Press)

Perryman, M. A. C., Lindegren, L., Kovalevsky, J., et al. 1997, A\&A, 323, L49

Turon, C., \& Arenou, F. 2008, IAU Symp., 248, 1

van Leeuwen, F., \& Fantino, E. 2005, A\&A, 439, 791

Zacharias, N., Urban, S. E., Zacharias, M. I., et al. 2004, AJ, 127, 3043 